\title{
Left- and right-handed transmission peaks near the magnetic resonance frequency in composite metamaterials
}

\author{
N. Katsarakis, T. Koschny, and M. Kafesaki \\ Institute of Electronic Structure and Laser (IESL), Foundation for Research and Technology-Hellas (FORTH), P.O. Box 1527, \\ Vasilika Vouton, 71110 Heraklion, Crete, Greece \\ E. N. Economou \\ IESL-FORTH and Department of Physics, University of Crete, Heraklion, Crete, Greece \\ Ekmel Ozbay \\ Department of Physics, Bilkent University, Bilkent, 06533 Ankara, Turkey
}

C. M. Soukoulis*

IESL-FORTH and Department of Materials Science and Technology, University of Crete, Heraklion, Crete, Greece

(Received 6 February 2004; revised manuscript received 9 August 2004; published 5 November 2004)

\begin{abstract}
We present free-space microwave measurements on composite metamaterials (CMMs) consisting of split ring resonators (SRRs) and wires either on the same dielectric board or on alternating boards. Our experimental results disprove the widely held belief that the occurrence of a CMM transmission peak within the stop bands of the SRRs alone and wires alone constitutes a clear demonstration of left-handed (LH) behavior. This belief is based on the assumption that the stop bands of SRRs alone and wires alone are not affected by the simultaneous presence of both. We show here that this assumption is wrong: The effective plasma frequency, $\omega_{\mathrm{p}}^{\prime}$, of the CMM is actually substantially lower than the wires-only plasma frequency, $\omega_{\mathrm{p}}$; furthermore, the in-plane wires, as opposed to the off-plane case, push the magnetic resonance frequency of the SRRs, $\omega_{\mathrm{m}}$, to a higher value, $\omega_{\mathrm{m}}^{\prime}$, for the CMM. We conclude that the criterion for deciding whether a peak in the transmission spectrum through a CMM is really left-handed is for the peak to be located above $\omega_{\mathrm{m}}^{\prime}$ and below $\omega_{\mathrm{p}}^{\prime}$. Our results provide a definite way for experimentally identifying $\omega_{\mathrm{p}}^{\prime}$.
\end{abstract}

DOI: 10.1103/PhysRevB.70.201101

PACS number(s): 42.70.Qs, 41.20.Jb, 73.20.Mf

Left-handed metamaterials (LHMs) have attracted recently great attention due to their fascinating electromagnetic properties. It was Veselago who introduced the term "lefthanded substances" in his seminal work published in $1968 .{ }^{1}$ He suggested that in a medium with simultaneously negative permittivity, $\varepsilon$, and permeability, $\mu$, the index of refraction would be negative and the phase of the electromagnetic (EM) waves would propagate in a direction opposite to that of the EM energy flow. In this case, the vectors $\mathbf{k}, \mathbf{E}$, and $\mathbf{H}$ form a left-handed set and therefore Veselago referred to such materials as "left-handed." LHMs would exhibit peculiar electromagnetic properties such as the reversal of Snell's law, Doppler effect, and Čerenkov radiation. Nevertheless, Veselago's prediction did not attract much attention in the scientific community until very recently, since there was no experimental realization of materials with both $\varepsilon$ and $\mu$ negative over a certain frequency range.

The interest in Veselago's work was renewed since Pendry et al. proposed a structure consisting of nonmagnetic conducting elements embedded in a dielectric medium; ${ }^{2}$ this structure, which was called a split ring resonator (SRR), exhibited a resonant magnetic response resulting in a negative effective permeability, $\mu_{e f f}$, over a finite frequency range. Based on the proposal of Pendry et al. and targeting the original idea by Veselago, Smith et al. demonstrated in 2000 the realization of the first LHM consisting of alternating layers of SRRs and continuous wires, the latter being respon- sible for the negative $\varepsilon .{ }^{3}$ They were also able to fabricate a two-dimensional LHM based on a standard printed circuit board lithographic process. ${ }^{4}$ Since the original microwave experiment by Smith et al., ${ }^{3}$ several CMMs, composed of SRRs and wires, were fabricated that exhibited a passband in which it was thought that both $\varepsilon$ and $\mu$ were negative. ${ }^{5,6}$ This assumption was based on transmission measurements of the wires alone, the SRRs alone and the CMM. The occurrence of a CMM transmission $(T)$ peak within the stop bands of the SRRs-only and wires-only structures was taken as evidence for LH behavior. Besides the experiments, there is also a large amount of numerical work in which transmission and reflection data are calculated for a finite length of a $\mathrm{CMM}^{7-10}$ Using these data a retrieval procedure can be applied to obtain the metamaterial parameters $\varepsilon$ and $\mu$, with the assumption that the CMM can be treated as a homogeneous medium. This procedure confirmed ${ }^{11}$ that a medium composed of SRRs and wires can indeed be characterized by effective $\varepsilon$ and $\mu$ whose real parts are both negative over a finite frequency band.

However, it was recently found that the SRRs, in addition to their resonant magnetic response at $\omega_{\mathrm{m}}$, exhibit a resonant electric response at $\omega_{0},{ }^{12}$ which is similar to the electric response of a system of cut wires (wires of finite length). This electric response, added to the electric response of the continuous wires of the CMM, results in a new effective plasma frequency, $\omega_{\mathrm{p}}^{\prime}$, of the CMM, which is considerably lower 


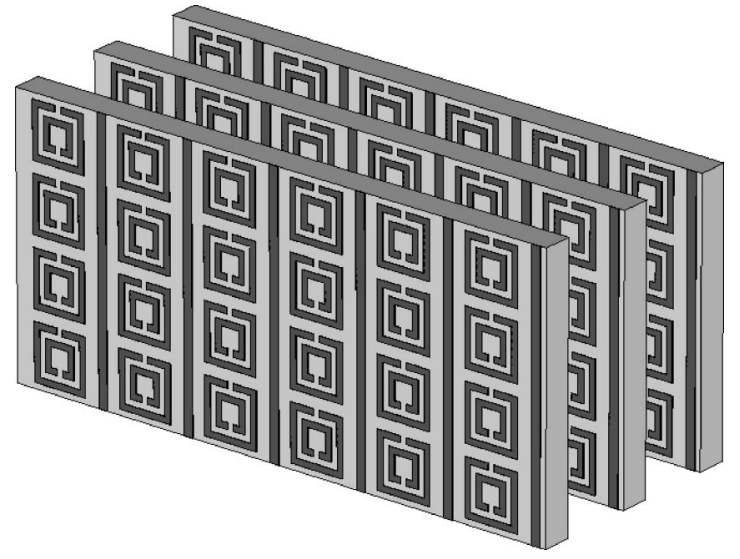

FIG. 1. Schematic drawing of an in-plane CMM composed of SRRs and continuous wires on the same side of the dielectric board.

than the plasma frequency of the wires, $\omega_{\mathrm{p}}$. This effect can be demonstrated by closing the SRRs (removing the gaps inside the rings), thus switching off the magnetic resonant response of the system and preserving only its total electric response. Based on the above findings, an easy to apply criterion was proposed ${ }^{12}$ to identify if an experimental transmission peak is LH or right-handed (RH): If the closing of the gaps of the SRRs in a given CMM removes only a single peak from the $T$ data (in the low-frequency regime), this is strong evidence that this $T$ peak is indeed LH. ${ }^{13}$ This criterion could become valuable in experimental studies, where one cannot easily obtain the effective $\varepsilon$ and $\mu$.

In this work we present free-space microwave measurements on CMMs consisting of SRR and wire structures, either on the same dielectric board or on alternating boards. It is demonstrated that the occurrence of a CMM transmission peak within the stop bands of the individual SRR and wire structures cannot be taken as evidence for LH behavior as it was thought before. We show that the electric response of the SRRs, ${ }^{12}$ added to the electric response of the continuous wires, results in a new effective plasma frequency, $\omega_{\mathrm{p}}^{\prime}$, of the CMM, which is considerably lower than the plasma frequency of the wires alone, $\omega_{\mathrm{p}}$. Moreover, our data show that the in-plane wires push the magnetic resonance frequency of the SRRs, $\omega_{\mathrm{m}}$, to a higher value, $\omega_{\mathrm{m}}^{\prime}$, for the CMM. Thus, we show that if a peak in the transmission spectrum is located between $\omega_{\mathrm{m}}^{\prime}$ and $\omega_{\mathrm{p}}^{\prime}\left(\omega_{\mathrm{m}}^{\prime}<\omega_{\mathrm{p}}^{\prime}\right)$, then it is really left-handed.

The CMMs studied here, consisting of SRRs and continuous wires, were fabricated using a conventional printed circuit board process, with copper patterns on $1.6 \mathrm{~mm}$ thick FR-4 dielectric substrates. The FR-4 board has a dielectric constant of $\sim 4.8$ and a dissipation factor of $\sim 0.017$ at $1.5 \mathrm{GHz}$.

First, CMMs with SRRs and wire structures on the same side of the boards were fabricated (in-plane CMMs, see Fig. 1). The geometry of the unit cell (u.c.) is shown in the inset of Fig. 2. The u.c. dimensions are $\alpha_{\mathrm{k}}=5 \mathrm{~mm}, \alpha_{\mathrm{E}}=3.63 \mathrm{~mm}$, and $\alpha_{\mathbf{H}}=5.6 \mathrm{~mm}$ and $6.3 \mathrm{~mm}$, and it contains one copper wire per SRR. The width of the copper wire is $0.5 \mathrm{~mm}$ while its thickness is $0.03 \mathrm{~mm}$. The SRR geometry is the same as in Ref. 5 and is described in Ref. 14. The periodicity along the $\mathbf{H}$ direction was obtained by stacking the boards. The

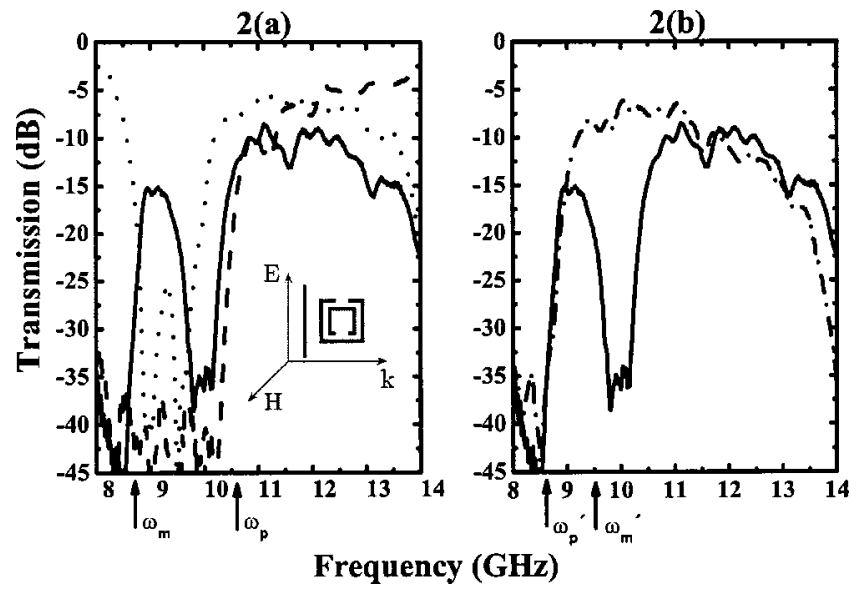

FIG. 2. (a) Measured transmission spectra for in-plane CMMs composed of SRRs and continuous wires (solid line), SRR-only structures (dotted line), and wires-only structures (dashed line). The latter two identify $\omega_{\mathrm{m}}$ and $\omega_{\mathrm{p}}$. The width of the continuous wires is $0.5 \mathrm{~mm}$. The inset shows the unit cell of the in-plane CMM (SRRs plus wires) as well as the electric field orientation and polarization; (b) in-plane CMM solid curve redrawn to show that the same threshold is exhibited (at $\omega_{\mathrm{p}}^{\prime}$ ) as in the nonmagnetic structure consisting of closed-SRRs and wires (dotted-dashed line). Thus the transmission peak in the CMM curve is actually $\mathrm{RH}$ and not $\mathrm{LH}$; the dip in the CMM spectrum corresponds to the SRR stop band, shifted due to the presence of the wires.

total system consisted of $25 \times 25 \times 25$ u.c. Furthermore, variations of the above CMMs were fabricated, containing closed SRRs (SRRs without the gaps) in the place of SRRs, as well as only SRRs and only wires.

The transmission of EM waves through in-plane CMMs, SRRs-only and wires-only structures, and finally CMMs containing SRRs with no gaps was measured. The transmission measurements were performed in free space, using a Hewlett-Packard 8722 ES network analyzer and microwave standard-gain horn antennas. For all measurements the wave propagation was parallel to the boards with the $\mathbf{E}$ polarization parallel to the wires and the continuous sides of the SRRs (see the inset of Fig. 2).

Figure 2(a) shows the measured $T$ spectra of SRRs-only structure (dotted line), wires-only structure (dashed line), and of the in-plane CMM (solid line), for $\alpha_{\mathbf{H}}=5.6 \mathrm{~mm}$. The SRRs-only structure shows the expected $T$ dip at $\sim 8.5-10 \mathrm{GHz}$, corresponding to the magnetic resonance of the SRR, while the wires-only structure shows a cutoff frequency at $\sim 10.5 \mathrm{GHz}$ that corresponds to its plasma frequency, $\omega_{\mathrm{p}}$. The CMM (solid line) shows a $T$ peak between 8.5 and $9.5 \mathrm{GHz}$ (as in Ref. 5), i.e., within the frequency region of the SRRs dip. The occurrence of a CMM transmission peak within the stop bands of the SRR-only and wiresonly structures was originally taken as clear evidence for the appearance of LH behavior. ${ }^{4,5}$ The fault with this reasoning is that it ignores the effects of the SRRs on the electric response of the wires. We demonstrate here experimentally these effects by closing the gaps of the SRRs, as suggested in Ref. 12. Indeed, by closing the gaps of the SRRs in the CMM we expect the magnetic resonance of the SRRs to be switched off and thus to really monitor only the electric re- 
sponse of the CMM. The changes in the electric response of the CMM from the closing of the gaps are expected to be weak, since only a small amount of metal is added (in the gaps of the SRRs), while the symmetry is preserved.

As can be clearly seen from Fig. 2(b), the $T$ spectrum for the metamaterial with closed SRRs (dotted-dashed line) is almost the same with that of the ordinary CMM (solid line), with the main difference being the absence of the dip at $\sim 10 \mathrm{GHz}$, which indicates that this dip is due to the SRR magnetic response. Moreover, it is observed that, as it was suggested in Ref. 12, the combination of the wires with the SRRs shifts the effective plasma frequency, $\omega_{\mathrm{p}}^{\prime}$, of the CMM to $\sim 8.5 \mathrm{GHz}$, which is lower than the plasma frequency of the wires-only array $\left(\omega_{\mathrm{p}} \sim 10.5 \mathrm{GHz}\right)$. Hence, we must conclude that in the CMM $\varepsilon>0$ for $f>8.5 \mathrm{GHz}$; consequently, there should be no LH peak for $f>8.5 \mathrm{GHz}$. Therefore, the peak observed at $\sim 9 \mathrm{GHz}$ ought to be RH. On the other hand, based on the same arguments one should expect a dip and not a peak at $\sim 9 \mathrm{GHz}$, since in this region $\varepsilon>0$ and $\mu<0$ (due to the SRR resonance). To explain this apparent discrepancy we examined the influence of the wires on the magnetic resonance frequency, $\omega_{\mathrm{m}}$, of the SRRs. We revealed numerically, using finite difference time domain calculations, ${ }^{15}$ that the currents in the wires tend, on the average, to diminish the magnetic flux on the SRRs and thus to decrease their effective inductance $\left(L_{\text {eff }}\right)$; the net result is an increase of the magnetic resonance frequency, since it is given by $1 /\left(L_{\text {eff }} C_{\text {eff }}\right)^{1 / 2}$ ( $C_{\text {eff }}$ is the effective capacitance). In our case, this led to a shift of the resonance frequency from $\omega_{\mathrm{m}} \sim 8.5 \mathrm{GHz}$ to $\omega_{\mathrm{m}}^{\prime} \sim 9.5 \mathrm{GHz}$, explaining the peak at $\sim 9 \mathrm{GHz}$ as $\mathrm{RH}$ and accounting for the dip in the CMM $T$ spectrum at $\sim 10 \mathrm{GHz}$ (as $\varepsilon>0$ and $\mu<0$ ). To further check this interpretation we placed the wires off-plane and symmetrically with respect to the SRRs (see the inset of Fig. 4). In this case the currents in the wires have no net effect on the magnetic flux in the SRRs, and consequently we expect $\omega_{\mathrm{m}}$ not to be influenced by the wires. Our microwave measurements for off-plane CMM structures confirmed this expectation.

For obtaining a real LH peak it is necessary $\omega_{\mathrm{p}}^{\prime}$ to be larger than $\omega_{\mathrm{m}}^{\prime}$. To achieve this we doubled the width of the copper wires to $1 \mathrm{~mm}$. The $T$ data for the newly developed in-plane CMM are shown in Figs. 3(a) and 3(b). It is shown that $\omega_{\mathrm{p}}^{\prime}$ is now at $\sim 10.4 \mathrm{GHz}$ [dotted-dashed line in Fig. $3(\mathrm{~b})]$. In this case $\omega_{\mathrm{p}}^{\prime}$ is clearly higher than $\omega_{\mathrm{m}}^{\prime}$ and thus the observed CMM peak [see solid line in Figs. 3(a) and 3(b)], centered at $\sim 9.5 \mathrm{GHz}$, is really $\mathrm{LH}$.

We finally tried to improve the transmittance of the $\mathrm{LH}$ peak by considering off-plane CMMs (theoretical results indicate higher transmittance for off-plane than for in-plane cases). In this case, the wires are printed on the back side of the boards opposite to the gaps of the SRRs. The unit cell for this case is shown in the inset of Fig. 4; its dimensions are $\alpha_{\mathbf{k}}=5 \mathrm{~mm}, \alpha_{\mathbf{E}}=3.63 \mathrm{~mm}$, and $\alpha_{\mathbf{H}}=5.6 \mathrm{~mm}, 3.1 \mathrm{~mm}$, and $2.6 \mathrm{~mm}$. The total system consists again of $25 \times 25 \times 25$ unit cells.

The $T$ spectrum for the off-plane CMM with $\alpha_{\mathbf{H}}$ $=3.1 \mathrm{~mm}$ is shown in Fig. 4. A well-defined peak can be clearly observed at $\sim 9.5 \mathrm{GHz}$. The closing of the gaps of the SRRs demonstrates that $\omega_{\mathrm{p}}^{\prime}$ is at $\sim 10.5 \mathrm{GHz}$. Since the

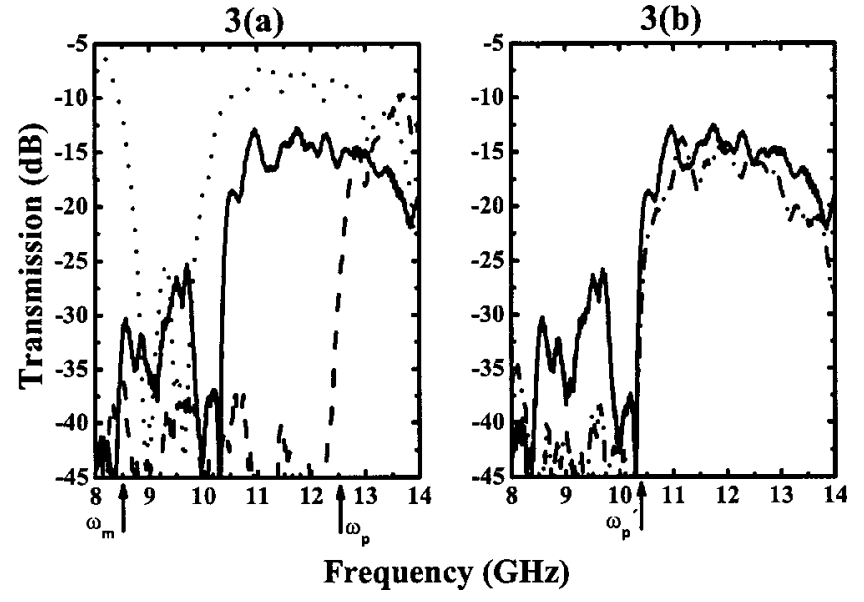

FIG. 3. (a) Measured transmission spectra for in-plane CMMs (solid line), SRRs-only structures (dotted line) and wires-only structures (dashed line). The width of the continuous wires has been increased to $1 \mathrm{~mm}$; (b) the coincidence of the CMM solid curve with the closed SRRs plus wires (dotted-dashed) curve determines the effective plasma frequency, $\omega_{\mathrm{p}}^{\prime}$, which is now well above the CMM transmission peak, identified as left-handed.

SRRs-only $T$ dip is at $8.5-10 \mathrm{GHz}$, it is apparent that the observed peak is LH.

In conclusion, we have shown experimentally that the effective plasma frequency, $\omega_{\mathrm{p}}^{\prime}$, of the CMM composed of SRRs and continuous wires is lower than the wires-only plasma frequency, $\omega_{\mathrm{p}}$. We have also demonstrated how to obtain experimentally an accurate value for $\omega_{\mathrm{p}}^{\prime}$. Furthermore, we showed that the in-plane wires, as opposed to the offplane configuration, push the magnetic resonance frequency, $\omega_{\mathrm{m}}$, to a slightly higher value, $\omega_{\mathrm{m}}^{\prime}$. Hence a peak in the EM wave transmission through a CMM is LH only if $\omega_{\mathrm{m}}^{\prime}<\omega_{\mathrm{p}}^{\prime}$

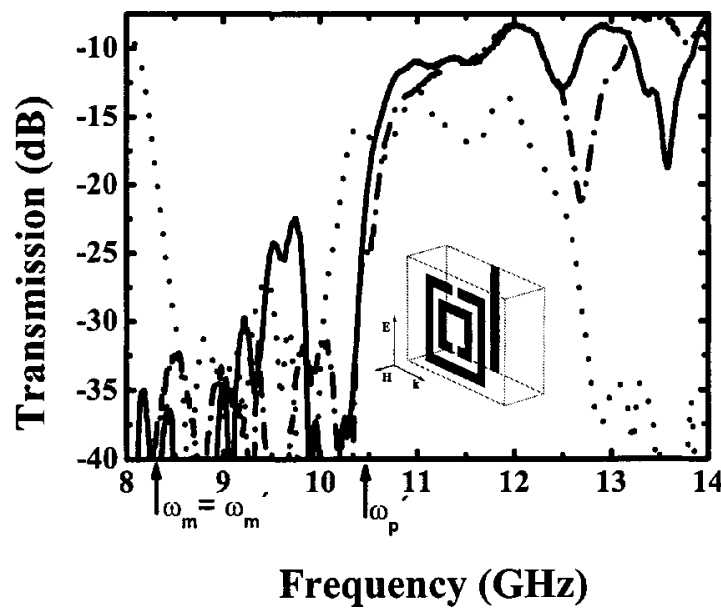

FIG. 4. Measured transmission spectra for off-plane CMMs composed of SRR and continuous wires (solid line), SRRs-only structures (dotted line) and structures consisting of closed SRRs and wires (dotted-dashed line). The width of the continuous wires is $1 \mathrm{~mm}$. The inset shows the unit cell of the off-plane CMM (SRRs plus wires) as well as the electric field orientation and polarization. The frequencies $\omega_{\mathrm{m}}=\omega_{\mathrm{m}}^{\prime}$ and $\omega_{\mathrm{p}}^{\prime}$ are well separated and the CMM peak is clearly LH. 
and if it lies between $\omega_{\mathrm{m}}^{\prime}$ and $\omega_{\mathrm{p}}^{\prime}$. In contrast, the condition $\omega_{\mathrm{m}}<\omega_{\mathrm{p}}$, which is widely used as a criterion for LH behavior, does not guarantee that an observed peak is LH, since the mutual interactions of wires and SRRs decrease the positive difference $\omega_{\mathrm{p}}-\omega_{\mathrm{m}}$ and possibly eliminate it altogether. To demonstrate these effects we presented microwave transmission measurements where $\omega_{\mathrm{m}}<\omega_{\mathrm{p}}$ but $\omega_{\mathrm{m}}^{\prime}>\omega_{\mathrm{p}}^{\prime}$ and hence the observed peak was $\mathrm{RH}$, as well as measurements where $\omega_{\mathrm{m}}^{\prime}<\omega_{\mathrm{p}}^{\prime}$ and the observed peak was LH.

This work was supported by Ames Laboratory (contract No. W-7405-Eng-82). Financial support of DARPA (contract No. MDA972-01-2-0016), NSF (U.S.-Greece Collaboration), and EU FET project DALHM are also acknowledged.
*Permanent address: Ames Laboratory and Dept. of Physics and Astronomy, Iowa State University, Ames, IA 50011.

${ }^{1}$ V. G. Veselago, Usp. Fiz. Nauk 92, 517 (1968) [Sov. Phys. Usp. 10, 509 (1968)].

${ }^{2}$ J. B. Pendry, A. Holden, D. Robbins, and W. Stewart, IEEE Trans. Microwave Theory Tech. 47, 2075 (1999).

${ }^{3}$ D. R. Smith, W. J. Padilla, D. C. Vier, S. C. Nemat-Nasser, and S. Schultz, Phys. Rev. Lett. 84, 4184 (2000).

${ }^{4}$ R. A. Shelby, D. R. Smith, S. C. Nemat-Nasser, and S. Schultz, Appl. Phys. Lett. 78, 489 (2001).

${ }^{5}$ M. Bayindir, K. Aydin, E. Ozbay, P. Markoš, and C. M. Soukoulis, Appl. Phys. Lett. 81, 120 (2002).

${ }^{6}$ K. Li, S. J. McLean, R. B. Gregor, C. G. Parazzoli, and M. Tanielian, Appl. Phys. Lett. 82, 2535 (2003).

${ }^{7}$ T. Weiland, R. Schummann, R. B. Greegor, C. G. Parazzoli, A. M. Vetter, D. R. Smith, D. V. Vier, and S. Schultz, J. Appl. Phys. 90, 5419 (2001); P. Markoš and C. M. Soukoulis, Phys. Rev. E 65, 036622 (2002).

${ }^{8}$ P. Markoš, I. Rousochatzakis, and C. M. Soukoulis, Phys. Rev. E 66, 045601 (2002).
${ }^{9}$ J. Pacheco, Jr., T. M. Grzegorczyk, B.-I. Wu, Y. Zhang, and J. A. Kong, Phys. Rev. Lett. 89, 257401 (2002).

${ }^{10}$ P. Markoš and C. M. Soukoulis, Opt. Express 11, 649 (2003); P. Markoš and C. M. Soukoulis, Opt. Lett. 28, 846 (2003).

${ }^{11}$ D. R. Smith, S. Schultz, P. Markoš, and C. M. Soukoulis, Phys. Rev. B 65, 195104 (2002); T. Koschny, P. Markoš, D. R. Smith, and C. M. Soukoulis, Phys. Rev. E 68, 065602 (2003).

${ }^{12}$ T. Koschny, M. Kafesaki, E. N. Economou, and C. M. Soukoulis, Phys. Rev. Lett. 93, 107402 (2004).

${ }^{13}$ The criterion is valid if the closing of the SRR gaps preserves the SRR symmetry in respect to the electric field, i.e., when the parallel to the electric field sides of the SRR remain unchanged by the closing of the gaps. This is the case for all structures studied here.

${ }^{14}$ For the SRRs fabricated within this work the ring width, ring distance, ring separation, and the gaps of the rings are all $0.33 \mathrm{~mm}$. The larger SRR side is $3 \mathrm{~mm}$ and the thickness of the metal is $30 \mu \mathrm{m}$.

${ }^{15}$ R. W. Ziolkowski, Opt. Express 11, 662 (2003). 